\title{
SLC25A10 performs an oncogenic role in human osteosarcoma
}

\author{
GAOYUAN WANG $^{1}$, JIANJUN XIA ${ }^{2}$, CHENG CHEN $^{3}$, JIE QIU $^{1}$, PO SUN $^{1}$, \\ ZHIWEI PENG ${ }^{1}$, XIAOYU CHEN ${ }^{4}$ and BIN XU ${ }^{1}$ \\ ${ }^{1}$ Department of Orthopaedics, The First Affiliated Hospital of Anhui Medical University, Hefei, Anhui 230022; ${ }^{2}$ Department of \\ Orthopaedics, East District of The First Affiliated Hospital of Anhui Medical University, Hefei, Anhui 231600; \\ ${ }^{3}$ Department of Orthopaedics, People's Hospital of Fuyang City, Fuyang, Anhui 236015; ${ }^{4}$ Department \\ of Histology and Embryology, Anhui Medical University, Hefei, Anhui 230023, P.R. China
}

Received July 31, 2019; Accepted April 15, 2020

DOI: 10.3892/ol.2020.11863

\begin{abstract}
Osteosarcoma is one of the most common primary malignant bone tumors in adolescents. It is associated with high risk of relapse and the outcomes of patients with high-grade osteosarcoma remain poor. Therefore, additional studies investigating the molecular mechanisms involved in tumor initiation, growth, migration and invasion of osteosarcoma are necessary. In the present study, the protein levels of solute carrier family 25 member 10 (SLC25A10) were increased in osteosarcoma tissue, compared with normal bone tissue. In patients with osteosarcoma, high expression levels of SLC25A10 were associated with poor clinicopathological parameters, including metastasis, clinical Enneking stage, relapse-free survival and overall survival rates. Short hairpin RNA knockdown of SLC25A10 significantly suppressed cell proliferation as determined by cell counting, MTT assay and cell colony formation assays. In addition, SLC25A10 knockdown caused an increase in apoptosis and a decrease in mitosis in osteosarcoma cells. Cyclin E1 (CCNE1) was positively regulated by SLC25A10, while P21 and P27 were negatively regulated by SLC25A10. Therefore, SLC25A10 may play an oncogenic role in human osteosarcoma, which could be mediated by CCNE1, P21 and P27.
\end{abstract}

Correspondence to: Professor Xiaoyu Chen, Department of Histology and Embryology, Anhui Medical University, 81 Meishan Road, Hefei, Anhui 230023, P.R. China

E-mail: 13866700471@163.com

Professor Bin Xu, Department of Orthopaedics, The First Affiliated Hospital of Anhui Medical University, 218 Jixi Road, Hefei, Anhui 230022, P.R. China

E-mail: xubinyouchen100@126.com

Abbreviations: SLC25A10, solute carrier 25 family member 10; RFS, relapse-free survival; OS, overall survival; shRNA, short hairpin RNA; PARP, poly ADP-ribose polymerase

Key words: SLC25A10, proliferation, apoptosis, mitosis, osteosarcoma

\section{Introduction}

Osteosarcoma mainly arises from human mesenchymal cells and is one of the most common primary malignant bone tumors (1-3). The incidence of osteosarcoma in adolescents is relatively high $(4,5)$. The main treatment option for low-grade osteosarcoma is surgery alone, and the prognosis for patients with low-grade osteosarcoma is relatively good. High-grade osteosarcoma or osteosarcoma with metastasis can be treated with surgery and chemotherapy; however, the mean 5-year survival rate is $<25 \%(1,6,7)$. Although the treatment for osteosarcoma has improved, few effective methods are available $(8,9)$. Research has focused on the mechanisms involved in the initiation and development of osteosarcoma, yet the intrinsic mechanisms involved in the malignant behaviors of osteosarcoma remain unclear. Further studies are needed to improve the understanding of the molecular mechanisms involved in osteosarcoma initiation, growth, migration and invasion, which could help the development of novel treatments for osteosarcoma.

Solute carrier 25 family member 10 (SLC25A10), also known as dicarboxylate carrier, is an important regulator of human energy metabolism and redox homeostasis $(10,11)$. SLC25A10 contains six transmembrane regions and three cognate repeats, and is localized in the cytoplasm and the inner mitochondrial membrane $(12,13)$. SLC25A10 transports dicarboxylate substrates and supplies substrates for several pathways, including sulfur metabolism and gluconeogenesis $(10,14)$. SLC25A10 is involved in fatty acid synthesis, glucose-stimulated insulin secretion and additional physiological processes $(12,15,16)$. Moreover, Zhou et al (10) suggested that knockdown of SLC25A10 in human cancer cells markedly decreased cell growth and increased sensitivity to anticancer drugs, demonstrating its role as an oncogene. However, the role of SLC25A10 in different types of human cancer, including osteosarcoma, remains unclear. Therefore, further studies focusing on osteosarcoma are required.

The present study demonstrated that the expression levels of SLC25A10 were higher in human osteosarcoma tissues, compared with normal bone tissues. In addition, in patients with osteosarcoma, the expression levels of SLC25A10 were positively associated with tumor metastasis, clinical Enneking stage, poor relapse-free survival (RFS) and overall survival 
(OS) rates. Knockdown of SLC25A10 with short hairpin RNA (shRNA) significantly decreased cell proliferation, increased cell apoptosis and suppressed cell mitosis in osteosarcoma cells. Moreover, cyclin E1 (CCNE1) was positively regulated by SLC25A10, while P21/P27 were negatively regulated by SLC25A10. CCNE1 was previously described as an important tumor promoter in many types of human cancer, and P21/P27 were found to be tumor suppressors in many human cancer types (17-21). Collectively, CCNE1, P21 and P27 may mediate the oncogenic role of SLC25A10 in human osteosarcoma cells.

\section{Materials and methods}

Clinical osteosarcoma and normal bone samples. In total, 60 osteosarcoma tissues and 60 normal bone tissues were collected in The Department of Orthopedics and The Department of Pathology in The First Affiliated Hospital of Anhui Medical University. These tissues were collected from patients with osteosarcoma or bone diseases who underwent resection in The First Affiliated Hospital of Anhui Medical University between January 2011 and December 2013. These osteosarcoma tissues and normal bone tissues were not from the same patients. The clinicopathological features of the enrolled patients with osteosarcoma were collected from The Department of Pathology, The First Affiliated Hospital of Anhui Medical University. The 60 patients with osteosarcoma were followed-up for $>5$ years, and the RFS and OS rates were determined. Ethical approval from The Institutional Review Boards of Anhui Medical University was obtained prior to the study. All experiments involving human patients were performed according to The Code of Ethics of The World Medical Association (Declaration of Helsinki). Informed consent was obtained from all patients involved in the present study.

Immunohistochemistry. The protein levels of SLC25A10 in 4- $\mu \mathrm{m}$ thick paraffin sections of osteosarcoma tissues and normal bone tissues (10\% formalin fixed at room temperature for $24 \mathrm{~h}$ ) were detected by immunohistochemistry, as previously described $(22,23)$. Sections were deparaffinized in xylene, rehydrated in a series of ethanol solutions $(100,100,95,85$ and $75 \%)$ and heated in $0.01 \mathrm{M}$ sodium citrate buffer at $100^{\circ} \mathrm{C}$ for $10 \mathrm{~min}$ for antigen retrieval. Sections were incubated with $3 \%$ hydrogen peroxide incubation at room temperature for $10 \mathrm{~min}$, and then incubated with primary antibody [SLC25A10 rabbit polyclonal antibody (1:200; 12086-1-AP; ProteinTech Group, Inc.)] for $3 \mathrm{~h}$ at room temperature, followed by incubation for $15 \mathrm{~min}$ at room temperature with horseradish peroxidase (HRP)-conjugated secondary antibody (1:1; MaxVision-HRP, KIT-5030; Fuzhou Maixin Biotech Co., Ltd.) 3,3'-diaminobenzidine tetrahydrochloride (Fuzhou Maixin Biotech Co., Ltd.) was used for visualization. Sections with $<10 \%$ positive stained cells were considered as SLC25A10-negative, and sections with $\geq 10 \%$ positive stained cells were considered to be SLC25A10-positive using a light microscope (Olympus Corporation) at x20 magnification.

Cells and cell culture. The human MG-63 and U2OS osteosarcoma cell lines (both from American Type Culture Collection) were used in the present study. MG-63 and U2OS cells were cultured using DMEM medium (Gibco; Thermo Fisher
Scientific, Inc.) containing 10\% fetal bovine serum (Gibco; Thermo Fisher Scientific, Inc.) in humidified conditions at $37^{\circ} \mathrm{C}$ and $5 \% \mathrm{CO}_{2}$.

shRNA transfection. shRNAs targeting SLC25A10 (shSLC25A10-1 and shSLC25A10-2) and control shRNA (shCtrl) were used for cell functional experiments. All of the shRNAs were obtained from Shanghai GenePharma Co., Ltd. Lipofectamine ${ }^{\circledR} 2000$ (Invitrogen; Thermo Fisher Scientific, Inc.) was used for shRNA transfection ( $75 \mathrm{pmol} /$ transfection), which was performed as previously described $(22,23)$. The shRNA sequences were as follows: shSLC25A10-1, 5'-GTT TAGCTGGAGGCTTCGTGG-3'; shSLC25A10-2, 5'-CAA GCAGCTGGTCCTTAGCAC-3'; and shCtrl, 5'-TCAAGC TGCTAGGCCTATCCG-3'.

Western blotting. Western blotting experiments were performed to detect the protein expression levels of SLC25A10, CCNE1, P21 and P27 in human osteosarcoma cells. Western blot analysis was carried out as previously described $(22,23)$ and $\beta$-actin was used as a control. Polyvinylidene difluoridemembranes (EMD Millipore) were used for electrotransfer. Membranes were blocked using incubation with 5\% (w/v) non-fat milk powder at room temperature for $2 \mathrm{~h}$. Membranes were incubated with respective antibodies (rabbit polyclonal antibodies anti-SLC25A10 (1:1,000; 12086-1-AP; ProteinTech Group, Inc.), anti-CCNE1 (1:1,000; 11554-1-AP; ProteinTech Group, Inc.), anti-P21 (1:1,000; 10355-1-AP; ProteinTech Group, Inc.), anti-P27 (1:1,000; 25614-1-AP; ProteinTech Group, Inc.), rabbit polyclonal antibody anti-caspase-3 (1:500; \#9662; Cell Signaling Technology, Inc.), rabbit polyclonal anti-poly ADP-ribose polymerase (PARP; 1:500; \#9542; Cell Signaling Technology, Inc.) and rabbit polyclonal antibody anti- $\beta$-actin (1:1,000; 20536-1-AP; ProteinTech Group, Inc.) overnight at $4^{\circ} \mathrm{C}$. Then, then membranes were incubated with goat anti-rabbit IgG $(\mathrm{H}+\mathrm{L})$ HRP-conjugated secondary antibody $(1: 50,000 ; 31460$; Invitrogen; Thermo Fisher Scientific, Inc.) for $2 \mathrm{~h}$ at room temperature. Femto (34095; Thermo Fisher Scientific, Inc.) and Pico (34077; Thermo Fisher Scientific, Inc.) were used for visualization. Protein bands were identified using Image Quant LAS 4000 (Cytiva) and analyzed using ImageJ version 1.8.0 software (National Institutes of Health).

Cell proliferation assay. Cell counting, MTT and cell colony formation assays were performed to evaluate the role of SLC25A10 in the proliferation of human osteosarcoma cells, as previously described $(22,23)$. MG-63 and U2OS cells were transfected with shRNAs, and collected after $24 \mathrm{~h}$. In the cell counting assay, 10,000 cells/well were seeded into six-well plates, and the total cell number was calculated every day during a period of 5 days. In the MTT assay, 2,000 cells/well were seeded into 96-well plates, and the MTT detection was performed for 5 days (the purple formazan was dissolved in DMSO, and the absorbance was measured at $570 \mathrm{~nm}$ ). In the cell colony formation assay, 1,000 cells/well were seeded into six-well plates, and cell colony formation was examined after 10 days.

Flow cytometry. After transfection, flow cytometry was carried out to examine apoptosis and mitosis in MG-63 and 
U2OS cells. After transfection with shRNA for $72 \mathrm{~h}$, MG-63 or U2OS cells were collected, fixed in $70 \%$ ethanol solution at $4^{\circ} \mathrm{C}$ for $30 \mathrm{~min}$ and incubated with Annexin V-FITC (Beyotime Institute of Biotechnology) and propidium iodide (Sigma-Aldrich; Merck KGaA) for $30 \mathrm{~min}$ at room temperature. Flow cytometric analysis was performed to examine cell apoptosis. Cells were collected, fixed in $70 \%$ ethanol solution at $4^{\circ} \mathrm{C}$ for $30 \mathrm{~min}$ and incubated with propidium iodide and RNAse A for 30 min at room temperature. Subsequently, a flow cytometer (LSR II; BD Biosciences) was used to examine cell mitosis. Data were analyzed using FlowJo version 7.6 software (Tree Star, Inc.).

Statistical analysis. The data are presented as the mean, calculated from three independent experiments. SPSS 25.0 (IBM Corp) was used for statistical analysis. For cell functional experiments, the respective results were standardized by percentage. For cell functional experiments and flow cytometry, one-way ANOVA followed by Bonferroni or Tamhane post hoc tests were used. Immunohistochemical results were analyzed using Pearson's $\chi^{2}$ test. For RFS and OS analysis, Kaplan-Meier curves were calculated and log-rank test was used to analyze the statistical significance. $\mathrm{P}<0.05$ was considered to indicate a statistically significant difference.

\section{Results}

Clinical analysis of SLC25A10 expression in patients with osteosarcoma. In total, 60 paraffin-embedded osteosarcoma tissue samples and 60 paraffin-embedded normal bone tissue samples were collected. The protein levels of SLC25A10 in these tissues were detected by immunohistochemistry, and a positive signal of SLC25A10 was mainly detected in the cytoplasm. Among these 60 osteosarcoma tissues, 18 (30.0\%) were SLC25A10-negative and 42 (70.0\%) were SLC25A10-positive $(\mathrm{P}<0.05$; Table I). Among the 60 normal bone tissues, 32 (53.3\%) were SLC25A10-negative and $28(46.7 \%)$ were SLC25A10-positive (Table I). The representative SLC25A10 expression levels in osteosarcoma and normal bone tissues are presented in Fig. 1A. Therefore, the protein levels of SLC25A10 were higher in human osteosarcoma tissues, compared with normal bone tissues.

The association between SLC25A10 expression and various clinicopathological features and survival rates in the 60 patients with osteosarcoma was analyzed. Positive expression of SLC25A10 was found to be associated with a higher risk of tumor metastasis $(\mathrm{P}=0.037)$ and worse clinical Enneking stage $(\mathrm{P}=0.042)$ in the 60 patients with osteosarcoma (Table II). However, there was no significant statistical significance in SLC25A10 expression between patient groups of different age, sex or tumor size (all $\mathrm{P}>0.05$; Table II).

Moreover, these 60 patients with osteosarcoma were all followed-up for $>5$ years, and potential associations between SLC25A10 expression and RFS and OS rates were examined. Patients with osteosarcoma who displayed positive expression of SLC25A10 showed decreased OS and RFS rates, compared with patients with negative SLC25A10 expression (both $\mathrm{P}<0.05$; Fig. 1B and $\mathrm{C}$ ). Therefore, high expression of SLC25A10 was associated with poor prognosis in patients with osteosarcoma.
Table I. Expression of SLC25A10 in osteosarcoma and normal tissues.

\begin{tabular}{lccc}
\hline & & \multicolumn{2}{c}{ SLC25A10 expression } \\
\cline { 3 - 4 } Group & $\mathrm{n}$ & Negative, $\mathrm{n}(\%)$ & Positive, $\mathrm{n}(\%)$ \\
\hline Osteosarcoma & 60 & $18(30.0)$ & $42(70.0)^{\mathrm{a}}$ \\
Normal & 60 & $32(53.3)$ & $28(46.7)$ \\
\hline
\end{tabular}

${ }^{\mathrm{a}} \mathrm{P}<0.05 \cdot \chi^{2}=6.720$. SLC25A10, solute carrier 25 family member 10 .

Role of SLC25A10 in osteosarcoma cell proliferation. The human osteosarcoma cell lines MG-63 and U2OS were selected for cell functional experiments. Protein levels of SLC25A10 decreased after transfection with shSLC25A10-1 or shSLC25A10-2 compared with shCtrl in both MG-63 and U2OS cells (Fig. 2A). As determined by the cell counting assay (Fig. 2B and C), both shSLC25A10-1 and shSLC25A10-2 significantly reduced the total cell number, compared with shCtrl in both MG-63 and U2OS cells over 5 days (standardized by percentage: MG-63 shCtrl, 100\%; MG-63 shSLC25A10-1, 55.25\%; MG-63 shSLC25A10-2, 41.61\%; P<0.01; U2OS shCtrl, 100\%; U2OS shSLC25A10-1, 61.55\%; U2OS shSLC25A10-2, 46.29\%; $\mathrm{P}<0.01)$. Moreover, as detected by the MTT assay (Fig. 2D and E), cell proliferation decreased significantly during a period of 5 days in both MG-63 and U2OS cells after transfection with shSLC25A10-1 or shSLC25A10-2, compared with shCtrl (MTT assay at day 5, standardized by percentage: MG-63 shCtrl, 100\%; MG-63 shSLC25A10-1, 74.79\%; MG-63 shSLC25A10-2, 67.23\%; P<0.01; U2OS shCtrl, 100\%; U2OS shSLC25A10-1, 75.57\%; U2OS shSLC25A10-2, $68.70 \%$; $\mathrm{P}<0.01$ ). In addition, MG-63 shSLC25A10-1 and MG-63 shSLC25A10-2 cells showed decreased cell colony formation compared with MG-63 shCtrl cells (standardized by percentage: MG-63 shCtrl 100\%, MG-63 shSLC25A10-1 22.31\%, MG-63 shSLC25A10-2 20.77\%; P<0.01). Moreover, U2OS shSLC25A10-1 and U2OS shSLC25A10-2 cells also showed decreased colony formation compared with U2OS shCtrl cells (Standardized by percentage: U2OS shCtrl 100\%, U2OS shSLC25A10-1 33.56\%, U2OS shSLC25A10-2 28.64\%; $\mathrm{P}<0.01$; Fig. 2F and G). Collectively, SLC25A10 knockdown suppressed cell proliferation in human osteosarcoma cells.

Role of SLC25A10 in osteosarcoma cell apoptosis and mitosis. The role of SLC25A10 in apoptosis and mitosis of osteosarcoma cells was evaluated by flow cytometry. SLC25A10 shRNA knockdown significantly increased the percentage of apoptotic cells in both MG-63 and U2OS cells (MG-63 shCtrl, 6.97\%; MG-63 shSLC25A10-1, 15.82\%; MG-63 shSLC25A10-2, 16.84\%; P<0.01; U2OS shCtrl, 6.89\%; U2OS shSLC25A10-1, 16.23\%; U2OS shSLC25A10-2, 16.67\%; P<0.01; Fig. 3A and B). Moreover, transfection of shSLC25A10-1 or shSLC25A10-2 significantly increased the percentage of cells in $\mathrm{G}_{1}$ phase and decreased the percentage of cells in S phase in both MG-63 and U2OS cells (Fig. 3C and D). Therefore, the present data suggested that SLC25A10 promoted cell mitosis and suppressed cell apoptosis in human osteosarcoma cells. 
Table II. Association of SLC25A10 expression with clinicopathological parameters from patients with osteosarcoma.

\begin{tabular}{|c|c|c|c|c|c|}
\hline \multirow[b]{2}{*}{ Parameter } & \multirow[b]{2}{*}{$\mathrm{n}$} & \multicolumn{2}{|c|}{ SLC25A10 expression, n (\%) } & \multirow[b]{2}{*}{ P-value } & \multirow[b]{2}{*}{$\chi^{2}$} \\
\hline & & Negative & Positive & & \\
\hline \multicolumn{6}{|l|}{ Age, years } \\
\hline$\leq 20$ & 27 & $7(25.9)$ & $20(74.1)$ & 0.533 & 0.388 \\
\hline$>20$ & 33 & $11(33.3)$ & $22(66.7)$ & & \\
\hline \multicolumn{6}{|l|}{ Sex } \\
\hline Male & 37 & $11(29.7)$ & $26(70.3)$ & 0.954 & 0.003 \\
\hline Female & 23 & $7(30.4)$ & $16(69.6)$ & & \\
\hline \multicolumn{6}{|c|}{ Tumor size, cm } \\
\hline$\leq 5$ & 36 & $9(25.0)$ & $27(75.0)$ & 0.301 & 1.071 \\
\hline$>5$ & 24 & $9(37.5)$ & $15(62.5)$ & & \\
\hline \multicolumn{6}{|l|}{ Metastasis } \\
\hline No & 42 & $16(38.1)$ & $26(61.9)$ & 0.037 & 4.369 \\
\hline Yes & 18 & $2(11.1)$ & $16(88.9)$ & & \\
\hline \multicolumn{6}{|c|}{ Enneking stage } \\
\hline I-II & 28 & $12(42.9)$ & $16(57.1)$ & 0.042 & 4.133 \\
\hline III & 32 & $6(18.8)$ & $26(71.2)$ & & \\
\hline
\end{tabular}

SLC25A10, solute carrier 25 family member 10.
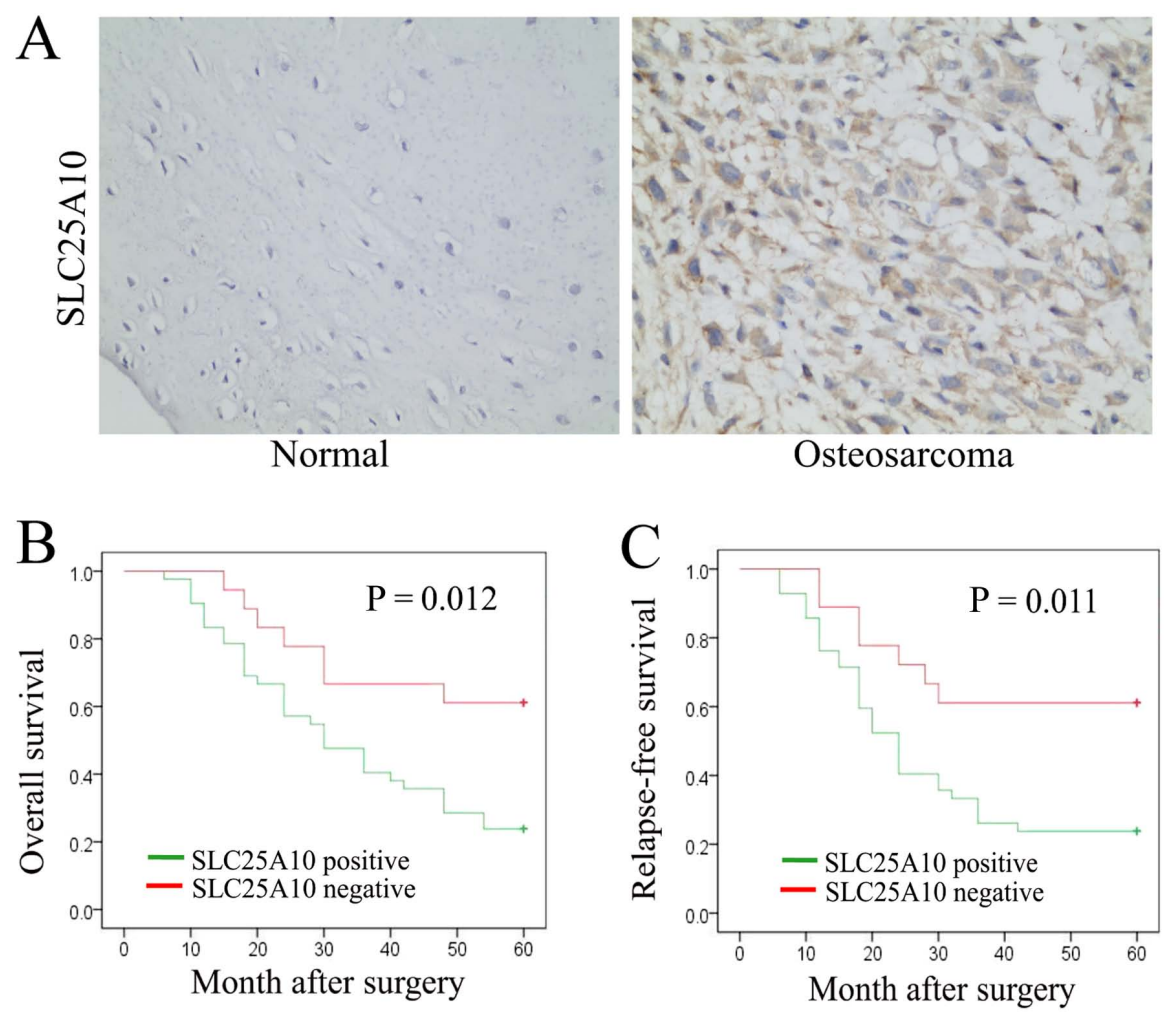

Figure 1. SLC25A10 expression in human osteosarcoma and normal bone tissues, and association between SLC25A10 expression and survival rates in patients with osteosarcoma. (A) Protein levels of SLC25A10 in 60 osteosarcoma tissues and 60 normal bone tissues were examined by immunohistochemistry. Magnification, x200. Kaplan-Meier curves were calculated to analyze (B) overall survival and (C) relapse-free survival rates between the SLC25A10-positive group and the SLC25A10-negative group in 60 patients with osteosarcoma. SLC25A10, solute carrier 25 family member 10.

Regulation analysis of SLC25A10 in osteosarcoma cells. To investigate the downstream mechanisms of SLC25A10, several candidate genes, including MYC, tumor protein 53 (TP53), cyclin D1 (CCND1), CCNE1, PTEN, STAT3, epidermal growth factor (EGFR), P21 and P27 were examined in MG-63 cells after transfection with shSLC25A10-1, shSLC25A10-2 

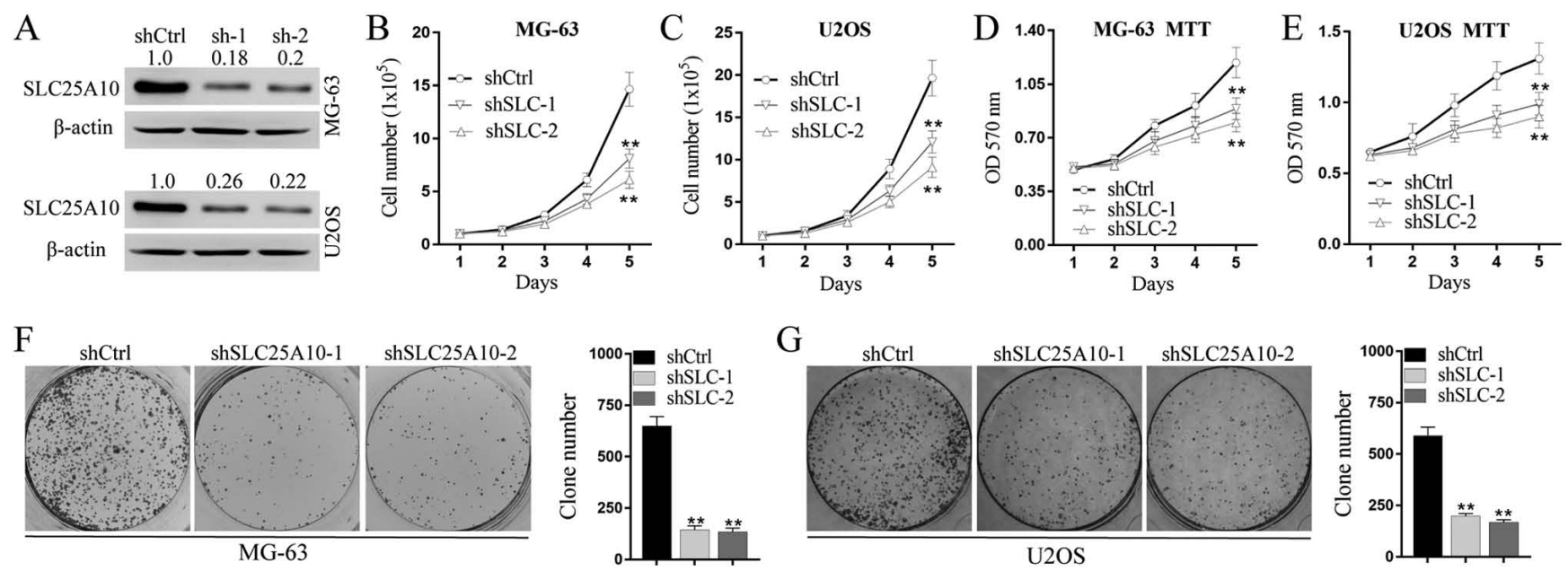

Figure 2. SLC25A10 promotes cell proliferation of human osteosarcoma cells. Osteosarcoma cells MG-63 and U2OS were transfected with shSLC25A10-1, shSLC25A10-2 or shCtrl. (A) Protein levels of SLC25A10 were examined by western blotting. $\beta$-actin was used as the loading control. Cell counting assays in (B) MG-63 and (C) U2OS cells were carried out and cell growth curves were calculated during a period of 5 days. MTT assays in (D) MG-63 cells and (E) U2OS cells were carried out and cell growth curves were calculated during a period of 5 days. Cell colony formation assays in (F) MG-63 cells and (G) U2OS cells were carried out and the cell colony number after 10 days were calculated. ${ }^{* *} \mathrm{P}<0.01 \mathrm{vs}$. shCtrl. sh, short hairpin; SLC25A10/SLC, solute carrier 25 family member 10; Ctrl, control; OD, optical density.

or shCtrl. These genes were selected according to previous studies which demonstrated their oncogenic role (24-31). Thus, it was hypothesized that these gene candidates were regulated by SLC25A10, and that they might mediate the role of SLC25A10 in osteosarcoma cells.

As determined by the western blotting assay, the protein level of CCNE1 decreased markedly after transfection with shSLC25A10-1 or shSLC25A10-2, whereas the protein levels of P21 and P27 increased after transfection with shSLC25A10-1 or shSLC25A10-2 in both MG-63 and U2OS cells, compared with shCtrl (Fig. 4A). However, there were no marked changes in MYC, TP53, CCND1, PTEN, STAT3 and EGFR protein levels after shSLC25A10 transfection compared with shCtrl (data not shown). Therefore, only CCNE1 was positively regulated by SLC25A10. Moreover, P21 and P27 were negatively regulated by SLC25A10 in human osteosarcoma cells.

In addition, cleaved caspase- 3 and its substrate cleaved PARP markedly increased after transfection with shSLC25A10-1 or shSLC25A10-2 in both MG-63 and U2OS cells. Therefore, knockdown of SLC25A10 promoted caspase-3-mediated apoptosis in human osteosarcoma cells.

\section{Discussion}

In the present study, several systematical experiments were performed to examine the role of SLC25A10 in human osteosarcoma. As examined by immunohistochemistry, the protein levels of SLC25A10 were much higher in osteosarcoma tissues compared with normal bone tissues. A high expression level of SLC25A10 was associated with worse clinicopathological parameters, including tumor metastasis and clinical Enneking stage in patients with osteosarcoma. Patients with osteosarcoma with high expression levels of SLC25A10 showed lower RFS and OS rates, compared with patients with low expression levels of SLC25A10. In MG-63 and U2OS human osteosarcoma cells, shRNA-mediated SLC25A10 knockdown significantly suppressed cell proliferation as determined by cell counting, MTT and colony formation assays. In addition, knockdown of SLC25A10 promoted apoptosis and suppressed mitosis in human osteosarcoma cells, as determined by flow cytometry. In addition, knockdown of SLC25A10 promoted caspase-3-mediated apoptosis. Zhou et al (10) demonstrated that SLC25A10-knockdown in A549 cells decreased cell proliferation, leading to a less malignant phenotype, as well as increased glutamine dependency and sensitivity to oxidative stress. The data from the present study are in line with these previous findings. However, few previous studies have documented the role of SLC25A10 in other human cancer types. Therefore, to the best of the authors' knowledge, the present study is the first to show that SLC25A10 may play a tumor-promoting role in human osteosarcoma.

In addition, the downstream pathway underlying the oncogenic role of SLC25A10 was examined, and CCNE1 was found to be positively regulated by SLC25A10. CCNE1 is a member of the cyclin family, which contributes to the activity of the cell cycle. As reported in previous study, CCNE1 is upregulated, and plays an oncogenic role in human osteosarcoma (17). MicroRNA (miR)-874 and miR-132 directly targeted CCNE1, and both acted as tumor suppressors, inhibiting the malignant features of human osteosarcoma cells. As a tumor promoter, CCNE1 mediated the tumor-suppressing effect of miR-874 and miR-132 (26,32). Moreover, CCNE1 was found to act as an oncogene in various other types of human cancer, including breast cancer $(18,33)$, lung cancer (34), gastric cancer (35) and hepatocellular carcinoma (36). Therefore, the tumor-promoting role of SLC25A10 in human osteosarcoma might be partly mediated by CCNE1.

Furthermore, in the present study, P21 and P27 were found to be negatively regulated by SLC25A10 in osteosarcoma cells. P21 is an important cyclin-dependent kinase inhibitor, which suppresses both cell proliferation and metastasis in human osteosarcoma cells. Moreover, overexpression of P21 increases sensitivity of osteosarcoma cells to anti-cancer drugs (37-39). Furthermore, P21 acts as tumor suppressor in 
A

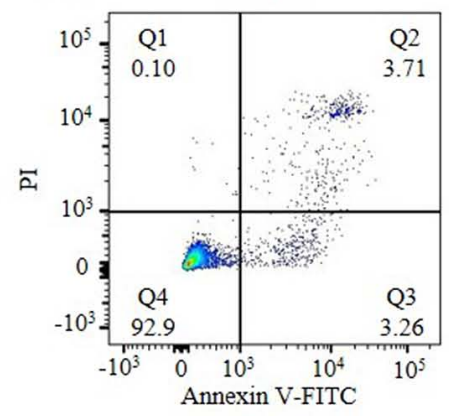

B

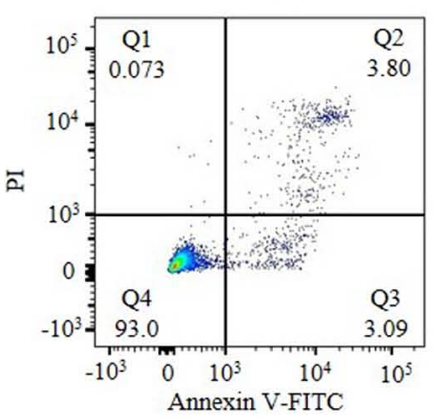

shSLC-1

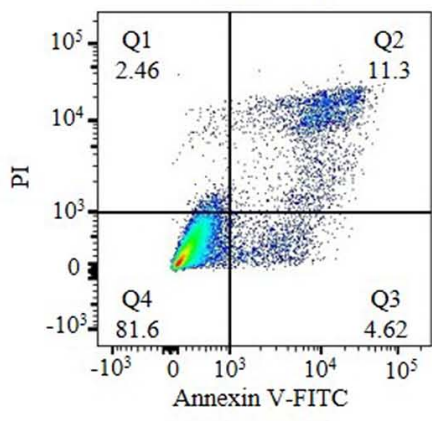

shSLC-1

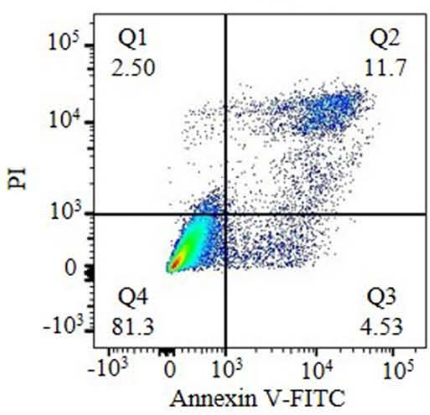

ShSLC-2
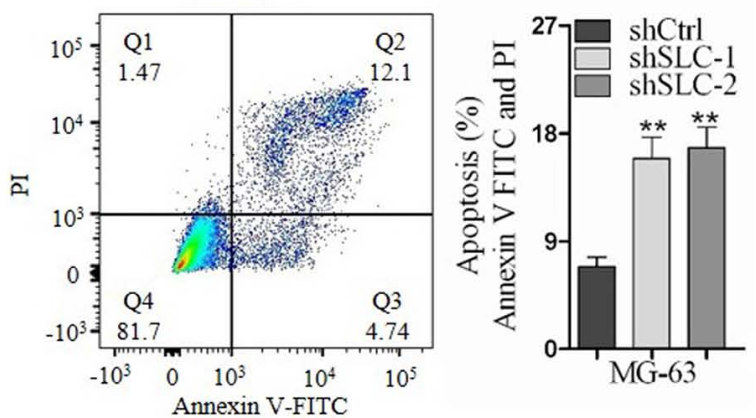

C

shCtrl

shSLC-1
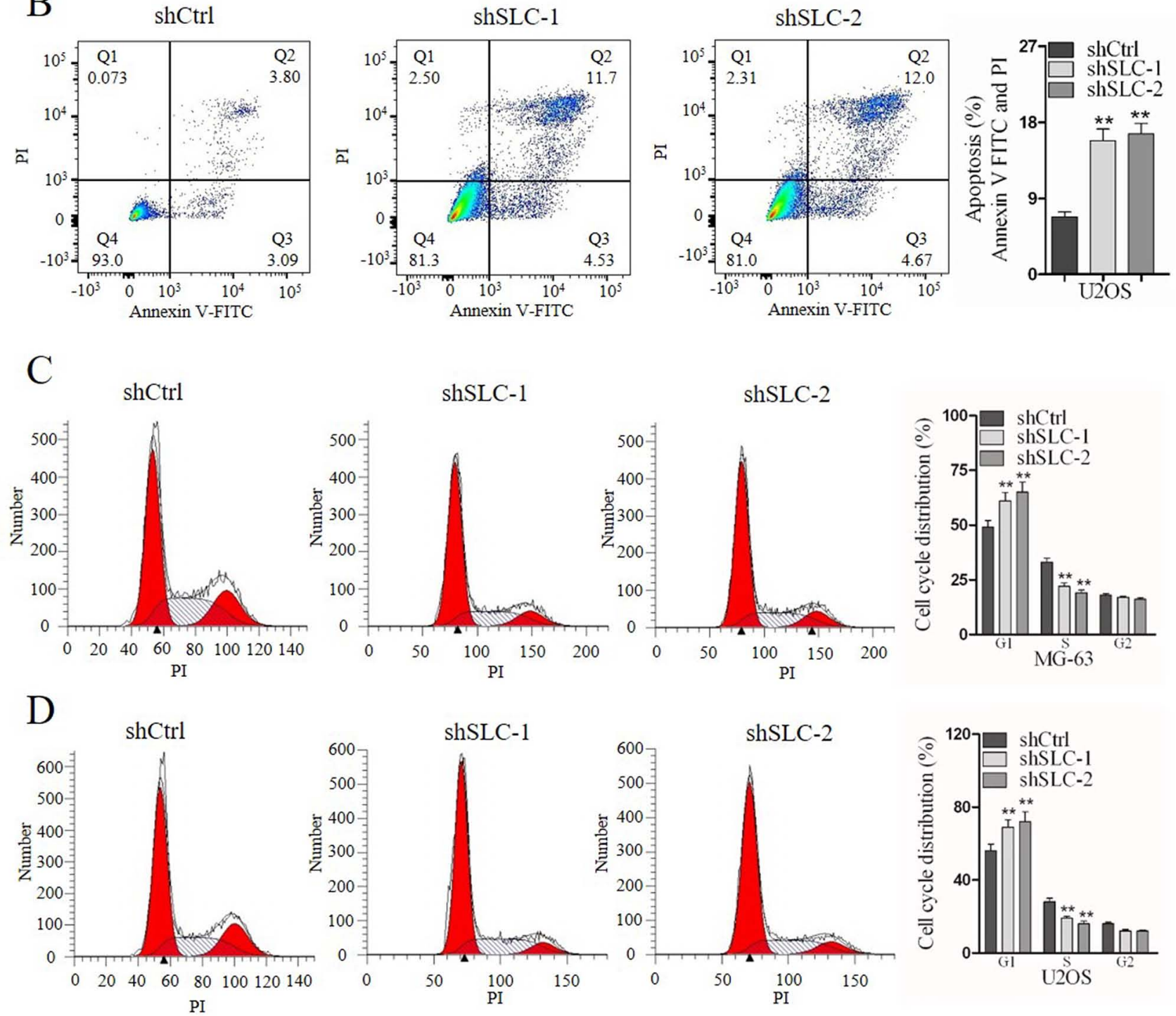

Figure 3. SLC25A10 knockdown promotes cell apoptosis and suppresses cell mitosis in human osteosarcoma cells. Osteosarcoma cells MG-63 and U2OS were transfected with shSLC25A10-1, shSLC25A10-2 or shCtrl. (A) Flow cytometry with Annexin V-FITC and PI staining in (A) MG-63 cells and (B) U2OS cells were performed to evaluate cell apoptosis. Flow cytometry with PI staining in (C) MG-63 cells and (D) U2OS cells were performed to evaluate the proportions of cells in the $\mathrm{G}_{1}, \mathrm{~S}$ and $\mathrm{G}_{2}$ phases. ${ }^{* *} \mathrm{P}<0.01$ vs. shCtrl. sh, short hairpin; SLC25A10/SLC, solute carrier 25 family member 10 ; PI, propidium iodide; Ctrl, control.

human nasopharyngeal carcinoma (19), cervical cancer (20), breast cancer (40), non-small cell lung cancer (41). P27 is a tumor suppressor gene in many types of human cancer. In osteosarcoma, Liao et al (42) identified that P27 is negatively correlated with S-phase kinase associated protein 2 and serves a tumor-suppressing role. Hu et al (43) demonstrated that inhibition of P27 mediated the tumor-promoting role of miR-227 in both cell proliferation and metastasis of 
A

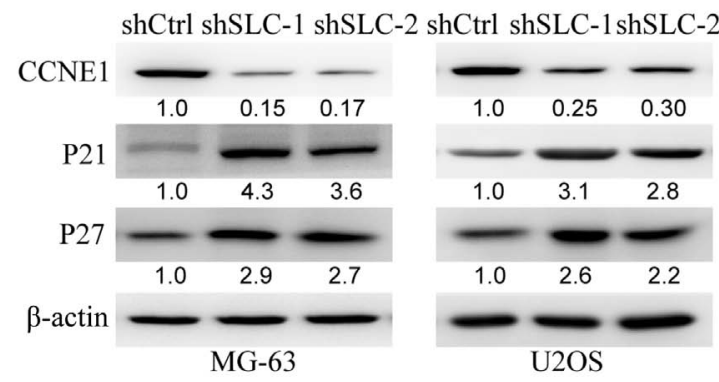

B
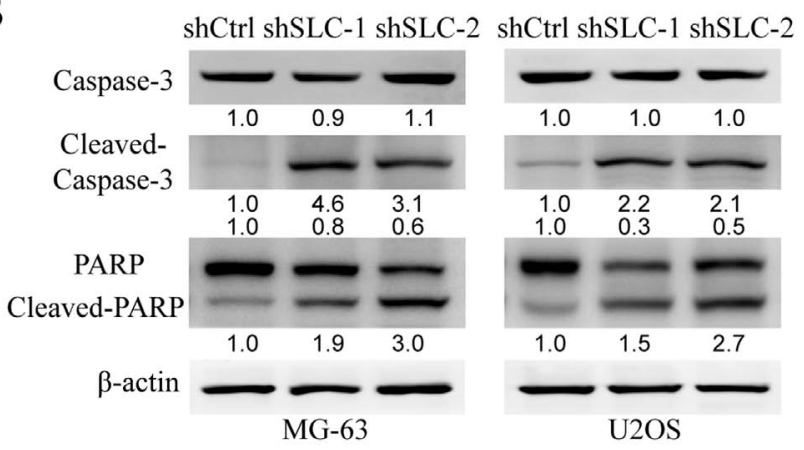

Figure 4. CCNE1, P21 and P27 are regulated by SLC25A10 in human osteosarcoma cells. (A) Protein levels of CCNE1, P21 and P27 in MG-63 and U2OS cells after transfection with shSLC25A10-1, shSLC25A10-2 or shCtrl were examined by western blot analysis. $\beta$-actin was used as loading control. The numbers below were fold change compared with reference protein. (B) Protein levels of caspase-3, cleaved caspase-3, PARP and cleaved PARP in MG-63 and U2OS cells following transfection with shSLC25A10-1, shSLC25A10-2 or shCtrl were examined by western blotting. The numbers below were fold change compared with reference protein respectively. sh, short hairpin; SLC25A10/SLC, solute carrier 25 family member 10; CCNE1, cyclin E1; PARP, poly ADP-ribose polymerase; Ctrl, control.

osteosarcoma cells. Accumulating evidence has demonstrated the tumor-suppressing role of P27 in human cervical cancer (20), pancreatic cancer (21), papillary thyroid cancer (44) and other types of cancer. Therefore, the downregulation of P21 and P27 increased by high expression levels of SLC25A10 in osteosarcoma cells may also mediate the tumor-promoting role of SLC25A10. The SLC25A10/CCNE1/P2/P27 signaling pathway may play an important role in human osteosarcoma.

Previous studies suggested that SLC25A10 was involved in sustaining metabolic shift and redox balance (10,13). Abnormal redox condition was found to be associated with abnormal expression of P21 and P27, thus influencing cell proliferation and mitosis $(45,46)$. CCNE1 is an important gene involved in cell cycle (47). Therefore, SLC25A10 may regulate the expression of CCNE1, P21 and P27 by influencing the redox state in osteosarcoma cells. Moreover, P21 may be regulated by SLC25A10. However, a previous study demonstrated that the upstream factor TP53 in the TP53/P21 pathway showed no significant changes after knockdown of SLC25A10 (48). Therefore, SLC25A10 may regulate $\mathrm{P} 21$ through TP53-independent mechanisms in osteosarcoma cells. In addition, knockdown of SLC25A10 may induce $\mathrm{G}_{1}$ cell cycle arrest by undetermined mechanisms, thus increasing the expression levels of P21, P27 and CCNE1. The changes in the levels of CCNE1, P21 and P27 could be an indirect consequence of cell cycle arrest. The exact molecular mechanisms underlying the regulation of CCNE1, P21 and P27 by SLC25A10 in osteosarcoma cells require further examination. The present study did not clarify the energy metabolism, redox homeostasis and the location of SLC25A10 in osteosarcoma cells, which was a limitation to the present study and should be investigated in future experiments.

In summary, the present study identified an oncogenic role for SLC25A10 in human osteosarcoma. In vitro functional experiments and analysis of tumor tissues collected from patients with osteosarcoma were performed. Decreased expression of SLC25A10 was associated with less malignant features in osteosarcoma cells and improved clinicopathological parameters and prognosis in patients with osteosarcoma. In addition, CCNE1 was positively regulated and P21/P27 were negatively regulated by SLC25A10. The present results suggested that the SLC25A10/CCNE1/P21/P27 pathway may be fundamental in human osteosarcoma.

\section{Acknowledgements}

Not applicable.

\section{Funding}

The present study was supported by grants from Anhui Province Natural Science Youth Funding Project (grant no. 1808085QH241) and Anhui Province Natural Science Funding Project (grant no. 1808085MH243).

\section{Availability of data and materials}

The datasets used and/or analyzed during the current study are available from the corresponding author on reasonable request.

\section{Authors' contributions}

GW, XC and BX designed experiments. GW, JX, CC and JQ performed experiments. GW, JX, PS and ZP analyzed data. XC and BX wrote the manuscript. All authors read and approved the final manuscript.

\section{Ethics approval and consent to participate}

The present study was approved by Institutional Review Boards of Anhui Medical University. All participants signed informed consent before admission.

\section{Patient consent for publication}

All patients signed informed consent for the possible publication of the present study.

\section{Competing interests}

The authors declare that they have no competing interests.

\section{References}

1. Fuja DG, Rainusso NC, Shuck RL, Kurenbekova L, Donehower LA and Yustein JT: Transglutaminase-2 promotes metastatic and stem-like phenotypes in osteosarcoma. Am J Cancer Res 8: 1752-1763, 2018. 
2. Abarrategi A, Tornin J, Martinez-Cruzado L, Hamilton A, Martinez-Campos E, Rodrigo JP, González MV, Baldini N, Garcia-Castro J and Rodriguez R: Osteosarcoma: Cells-of-Origin, cancer stem cells, and targeted therapies. Stem Cells Int 2016: 3631764, 2016.

3. Zhao X, Sun S, Xu J, Luo Y, Xin Y and Wang Y: MicroRNA-152 inhibits cell proliferation of osteosarcoma by directly targeting Wnt/B-catenin signaling pathway in a DKK1-dependent manner. Oncol Rep 40: 767-774, 2018.

4. He R, Wu JX, Zhang Y, Chen H and Yang L: LncRNA LINC00628 overexpression inhibits the growth and invasion through regulating PI3K/Akt signaling pathway in osteosarcoma. Eur Rev Med Pharmaco Sci 22: 5857-5866, 2018.

5. Nie WB, Zhao LM, Guo R, Wang MX and Ye FG: Circular RNA circ-NT5C2 acts as a potential novel biomarker for prognosis of osteosarcoma. Eur Rev Med Pharmaco Sci 22: 6239-6244, 2018.

6. Zuo DQ, Shogren KL, Zang J, Jewison DE, Waletzki BE, Miller AL II, Okuno SH, Cai Z, Yaszemski MJ and Maran A: Inhibition of STAT3 blocks protein synthesis and tumor metastasis in osteosarcoma cells. J Exp Clin Canc Res 37: 244, 2018.

7. Siegel RL, Miller KD and Jemal A: Cancer Statistics, 2016. CA Cancer J Clin 66: 7-30, 2016.

8. Tabak SA, Khalifa SE and Fathy Y: HER-2 Immunohistochemical Expression in Bone Sarcomas: A New hope for patients with osteosarcoma. Open Access Maced J Med Sci 6: 1555-1560, 2018

9. Meazza C and Scanagatta P: Metastatic osteosarcoma: A challenging multidisciplinary treatment. Expert Rev Anticancer Ther 16: 543-556, 2016.

10. Zhou XS, Paredes JA, Krishnan S, Curbo S and Karlsson A: The mitochondrial carrier SLC25A10 regulates cancer cell growth. Oncotarget 6: 9271-9283, 2015.

11. Zhao Q, Zhou XS, Curbo S and Karlsson A: Metformin downregulates the mitochondrial carrier SLC25A10 in a glucose dependent manner. Biochem Pharmacol 156: 444-450, 2018

12. Mizuarai S, Miki S, Araki H, Takahashi $\mathrm{K}$ and Kotani $\mathrm{H}$ Identification of dicarboxylate carrier Slc25a10 as malate transporter in de novo fatty acid synthesis. J Biol Chem 280 : 32434-32441, 2005.

13. Hlouschek J, Ritter V, Wirsdorfer F, Klein D, Jendrossek V and Matschke J: Targeting SLC25A10 alleviates improved antioxidant capacity and associated radioresistance of cancer cells induced by chronic-cycling hypoxia. Cancer Lett 439: 24-38, 2018.

14. Kulyte A, Ehrlund A, Arner P and Dahlman I: Global transcriptome profiling identifies KLF15 and SLC25A10 as modifiers of adipocytes insulin sensitivity in obese women. PLoS One 12: e0178485, 2017.

15. Huypens P, Pillai R, Sheinin T, Schaefer S, Huang M, Odegaard ML, Ronnebaum SM, Wettig SD and Joseph JW: The dicarboxylate carrier plays a role in mitochondrial malate transport and in the regulation of glucose-stimulated insulin secretion from rat pancreatic beta cells. Diabetologia 54: 135-145, 2011.

16. Lin Y, Berg AH, Iyengar P, Lam TK, Giacca A, Combs TP, Rajala MW, Du X, Rollman B, Li W, et al: The hyperglycemia-induced inflammatory response in adipocytes: The role of reactive oxygen species. J Biol Chem 280: 4617-4626, 2005.

17. Lockwood WW, Stack D, Morris T, Grehan D, O'Keane C, Stewart GL, Cumiskey J, Lam WL, Squire JA, Thomas DM and O'Sullivan MJ: Cyclin E1 is amplified and overexpressed in osteosarcoma. J Mol Diagn 13: 289-296, 2011.

18. ZhaoZM, Yost SE,Hutchinson KE, Li SM, Yuan YC, Noorbakhsh J, Liu Z, Warden C, Johnson RM, Wu X, et al: CCNE1 amplification is associated with poor prognosis in patients with triple negative breast cancer. BMC Cancer 19: 96, 2019.

19. Sang Y, Zhang R, Sun L, Chen KK, Li SW, Xiong L, Peng Y, Zeng L and Huang G: MORF4L1 suppresses cell proliferation, migration and invasion by increasing p21 and E-cadherin expression in nasopharyngeal carcinoma. Oncol Lett 17: 294-302, 2019

20. Guo Q, Xiong Y, Song Y, Hua K and Gao S: ARHGAP17 suppresses tumor progression and up-regulates P21 and P27 expression via inhibiting PI3K/AKT signaling pathway in cervical cancer. Gene 692: 9-16, 2019.

21. Li Z, Tao Y, Wang X, Jiang P, Li J, Peng M, Zhang X, Chen K, Liu H, Zhen P, et al: Tumor-secreted exosomal miR-222 promotes tumor progression via regulating P27 Expression and Re-localization in pancreatic cancer. Cell Physiol Biochem 51: 610-629, 2018

22. Chen CY, Lei J, Zheng Q, Tan S, Ding KS and Yu CJ: Poly(rC) binding protein 2 (PCBP2) promotes the viability of human gastric cancer cells by regulating CDK2. FEBS Open Bio 8: 764-773, 2018
23. Chen $\mathrm{C}$, Zheng Q, Kang $\mathrm{W}$ and $\mathrm{Yu} \mathrm{C}$ : Long non-coding RNA LINC00472 suppresses hepatocellular carcinoma cell proliferation, migration and invasion through miR-93-5p/PDCD4 pathway. Clin Res Hepatol Gastroenterol 43: 436-445, 2018

24. Wu X, Cai ZD, Lou LM and Zhu YB: Expressions of p53, c-MYC, BCL-2 and apoptotic index in human osteosarcoma and their correlations with prognosis of patients. Cancer Epidemiol 36: 212-216, 2012.

25. Cao W, Fang L, Teng S, Chen H and Liu T: MicroRNA-466 inhibits osteosarcoma cell proliferation and induces apoptosis by targeting CCND1. Exp Ther Med 16: 5117-5122, 2018.

26. Wang J, Xu G, Shen F and Kang Y: miR-132 targeting cyclin E1 suppresses cell proliferation in osteosarcoma cells. Tumour Biol 35: 4859-4865, 2014

27. Liu Q, Geng P, Shi L, Wang Q and Wang P: miR-29 promotes osteosarcoma cell proliferation and migration by targeting PTEN. Oncol Lett 17: 883-890, 2019.

28. Zhao R, He H, Zhu Y, Wan J, Li Y, Gao S and Zhang C: MiR-204/14-3-3 $\zeta$ axis regulates osteosarcoma cell proliferation through SATA3 pathway. Pharmazie 72: 593-598, 2017.

29. Wang J, Wang G, Li B, Qiu C and He M: miR-141-3p is a key negative regulator of the EGFR pathway in osteosarcoma. Onco Targets Ther 11:4461-4478, 2018.

30. Lv H, Gao G, Zhang L and Sun Y: Polo-like kinase 3 inhibits osteosarcoma cell proliferation and tumorigenesis via cooperative interaction with p21. Mol Med Rep 12: 6789-6796, 2015.

31. Zhang F and Peng H: LncRNA-ANCR regulates the cell growth of osteosarcoma by interacting with EZH2 and affecting the expression of p21 and p27. J Orthop Surg Res 12: 103, 2017.

32. Ghosh T,Varshney A,KumarP,KaurM,KumarV,ShekharR,DeviR, Priyanka P, Khan MM and Saxena S: MicroRNA-874-mediated inhibition of the major $\mathrm{G}_{1} / \mathrm{S}$ phase cyclin, CCNE1, is lost in osteosarcomas. J Biol Chem 292: 21264-21281, 2017.

33. Shukla K, Sharma AK, Ward A, Will R, Hielscher T, Balwierz A, Breunig C, Münstermann E, König R, Keklikoglou I and Wiemann S: MicroRNA-30c-2-3p negatively regulates NF- $\kappa$ B signaling and cell cycle progression through downregulation of TRADD and CCNE1 in breast cancer. Mol Oncol 9: 1106-1119, 2015.

34. Yao Y, Luo J, Sun Q, Xu T, Sun S, Chen M, Lin X, Qian Q, Zhang Y, Cao L, et al: HOXC13 promotes proliferation of lung adenocarcinoma via modulation of CCND1 and CCNE1. Am J Cancer Res 7: 1820-1834, 2017.

35. Zhang Y, Peng Z, Zhao YS and Chen L: microRNA-25 inhibits cell apoptosis of human gastric adenocarcinoma cell line AGS via regulating CCNE1 and MYC. Med Sci Monitor 22: 1415-1420, 2016.

36. Zhang X, Hu SJ, Zhang X, Wang L, Zhang X, Yan B, Zhao J, Yang A and Zhang R: MicroRNA-7 arrests cell cycle in G1 phase by directly targeting CCNE1 in human hepatocellular carcinoma cells. Biochem Biophys Res Commun 443: 1078-1084, 2014.

37. Fujii R, Osaka E, Sato K and Tokuhashi Y: MiR-1 suppresses proliferation of osteosarcoma cells by Up-regulating p21 via PAX3. Cancer Genom Proteom 16: 71-79, 2019.

38. Huang J, Deng GR, Liu TM, Chen WZ and Zhou Y: Long noncoding RNA PCAT-1 acts as an oncogene in osteosarcoma by reducing p21 levels. Biochem Biophys Res Commun 495: 2622-2629, 2018

39. Ding Y, Wang YC, Chen J, Hu Y, Cao Z, Ren P and Zhang Y: p21 overexpression sensitizes osteosarcoma U2OS cells to cisplatin via evoking caspase-3 and $\mathrm{Bax} / \mathrm{Bcl}-2$ cascade. Tumor Biol 35: 3119-3123, 2014.

40. Li Z, Yu D, Li H, Lv Y and Li S: Long noncoding RNA UCA1 confers tamoxifen resistance in breast cancer endocrinotherapy through regulation of the EZH2/p21 axis and the PI3K/AKT signaling pathway. Int J Oncol 54:1033-1042, 2019.

41. Guo L, Gu J, Hou S, Liu D, Zhou M, Hua T, Zhang J, Ge Z and $\mathrm{Xu}$ J: Long non-coding RNA DANCR promotes the progression of non-small-cell lung cancer by inhibiting p21 expression. Onco Targets Ther 12: 135-146, 2019.

42. Liao QD, Zhong D and Chen Q: Protein expression of Skp2 in osteosarcoma and its relation with prognosis. Zhong Nan Da Xue Xue Bao Yi Xue Ban 33: 606-611, 2008 (In Chinese).

43. Hu XH, Zhao ZX, Dai J, Geng DC and Xu YZ: MicroRNA-221 regulates osteosarcoma cell proliferation, apoptosis, migration, and invasion by targeting CDKN1B/p27. J Cell Biochem 120: 4665-4674, 2019.

44. Li H, Guan H, Guo Y, Liang W, Liu L, He X, Ke W, Cao X, $\mathrm{Xiao} \mathrm{H}$ and Li Y: CITED1 promotes proliferation of papillary thyroid cancer cells via the regulation of p21 and p27. Cell Biosci 8: 57, 2018 
45. Vijay K, Sowmya PR, Arathi BP, Shilpa S, Shwetha HJ, Raju M, Baskaran V and Lakshminarayana R: Low-dose doxorubicin with carotenoids selectively alters redox status and upregulates oxidative stress-mediated apoptosis in breast cancer cells. Food Chem Toxicol 118: 675-690, 2018

46. Zhang D, Wang Y, Liang Y, Zhang M, Wei J, Zheng X, Li F, Meng Y, Zhu NW, Li J, et al: Loss of p27 upregulates MnSOD in a STAT3-dependent manner, disrupts intracellular redox activity and enhances cell migration. J Cell Sci 127: 2920-2933, 2014.

47. Tang Z, Fang Y and Du R: MicroRNA-107 induces cell cycle arrests by directly targeting cyclin E1 in ovarian cancer. Biochem Biophys Res Commun 512: 331-337, 2019.
48. Yang M, Peng Y, Liu W, Zhou M, Meng Q and Yuan C: Sirtuin 2 expression suppresses oxidative stress and senescence of nucleus pulposus cells through inhibition of the p53/p21 pathway. Biochem Biophys Res Commun 513: 616-622, 2019.

This work is licensed under a Creative Commons Attribution-NonCommercial-NoDerivatives 4.0 International (CC BY-NC-ND 4.0) License. 